\title{
Reprodução assistida e relação conjugal durante a gravidez e após o nascimento do bebê: uma revisão da literatura
}

\author{
Isabela Machado da Silva \\ Rita de Cássia Sobreira Lopes \\ (Universidade Federal do Rio Grande do Sul)
}

\begin{abstract}
Resumo
A chegada de um bebê inicia um período de desafios e mudanças para o casal. Quando a gravidez é alcançada a partir da reprodução assistida, soma-se aos desafios já característicos desse período o impacto das experiências próprias a esse contexto. Neste trabalho, são revisadas e discutidas pesquisas que investigaram a relação de casais que recorreram à reprodução assistida, durante a gravidez e os dois primeiros anos do bebê. A revisão da literatura indica semelhanças entre esses casais e aqueles que conceberam naturalmente. No entanto, essas pesquisas tendem a basear-se na avaliação de variáveis específicas, como as relacionadas à satisfação conjugal e a características sociodemográficas, bem como pela ausência de um enfoque desenvolvimental que investigue a história prévia desse casal e suas famílias de origem. Esse enfoque, aliado a uma abordagem que privilegie a descrição das experiências desses casais, poderia contribuir para a compreensão desse contexto.
\end{abstract}

Palavras-chave: reprodução assistida; relação conjugal; gravidez; parentalidade.

\begin{abstract}
Assisted reproduction and marital relationship during the pregnancy and after the baby's birth: a literature review. A baby's arrival starts a period of challenges and changes for the couple. When pregnancy is achieved trough assisted reproduction, the impact of specific experiences related to this context is added to the already characteristic challenges of this period. In this article, the relationship of couples who were submitted to assisted reproduction during the pregnancy and the baby's first two years. A literature review indicates similarities between these couples and those who conceived naturally. However, these studies are based on the assessment of specific variables, such as marital satisfaction or adjustment, and socio-demographic variables. They are also characterized by the lack of a developmental perspective, which investigates the couple's previous history and the extended family. It is believed that this perspective, combined with an approach that emphasizes the couple's experiences description, could contribute for the comprehension of the context of assisted reproduction.
\end{abstract}

Keywords: assisted reproduction; marital relationship; pregnancy; parenthood.

A chegada de um filho apresenta, para o casal, uma série de novas demandas e prioridades, que exigem dos cônjuges uma reelaboração da imagem que fazem de si mesmos, do outro e do próprio relacionamento conjugal (Stern, 1997). Nesse processo de adaptação, importantes dimensões da relação do casal, tais como a intimidade, a comunicação e o sexo tendem a passar por modificações (Bradt, 1995), algumas das quais podem ser identificadas já na gravidez (Bornholdt, Wagner, \& Staudt, 2007; Lech \& Martins, 2003; Piccinini, Gomes, Nardi, $\&$ Lopes, 2008). Considera-se, assim, que a chegada de um filho é uma das transições que gera maior estresse na vida do casal (Pittman, 1987).

Autores como Belsky e Rovine (1990) defenderam, porém, que existe uma grande variabilidade na forma como cada casal reage a esse evento e afirmaram que é muito mais vantajoso investigar o que diferencia os casais do que apenas constatar tendências centrais. De acordo com estudos realizados, alguns fatores que influenciariam o impacto da chegada de um filho sobre a relação conjugal seriam as expectativas dos cônjuges (Delmore-Ko, Pancer, Hunsberger, \& Pratt, 2000), as experiências vivenciadas na família de origem (Perren, Wyl, Bürgin, Simoni, \& Klitzing, 2005), o planejamento da gravidez (Bouchard, Boudreau, \& Hébert, 2006) e os padrões de relacionamento adotados pelo casal até esse momento (Menezes, 2001). Dessa forma, esses estudos sugerem algumas questões a que os pesquisadores devem atentar ao investigar a relação conjugal durante esse período.

No entanto, nos casos em que os casais concebem com o auxílio das técnicas de reprodução assistida (TRA), deve-se considerar, ainda, a vivência de experiências específicas a esse 
contexto (Savitz-Smith, 2003). De acordo com Burns (2007), existem atualmente mais de 40 técnicas voltadas a auxiliar casais que não conseguem conceber naturalmente. Entre elas, encontram-se a inseminação artificial (IA), a fertilização in vitro (FIV), a injeção intracitoplasmática de espermatozóides (ICSI) e técnicas que envolvem a participação de terceiros, tais como doação de gametas e barriga de aluguel. No entanto, a utilização dessas técnicas tende a estar relacionada a eventos potencialmente estressantes (Hjelmstedt et al., 2006; McMahon, Gibson, Leslie, Cohen, \& Tennant, 2003): a constatação da infertilidade, a investigação de suas causas, a realização do tratamento e o grande investimento emocional presente em cada uma de suas etapas, que caracteriza uma fragmentação do processo de engravidar (Seger-Jacob, 2006).

Spotorno, Silva e Lopes (2008) corroboraram, em seu estudo, a idéia de que a infertilidade e seu tratamento constituem uma experiência complexa. Ao mesmo tempo em que são referidas ansiedade, frustração e a necessidade de ter que lidar com o sofrimento do marido e dos familiares, também se expressam sentimentos de esperança e otimismo, assim como um maior apoio por parte dos familiares e do próprio cônjuge. Borlot e Trindade (2004) destacaram, no entanto, que o efeito dessa experiência sobre o casal tende a ser influenciado pelas características anteriores de sua relação.

Pode-se perceber, portanto, que se trata de uma experiência marcada por um forte conteúdo emocional, o que leva autores como Burns (1987) a afirmarem que, independentemente do desfecho, os casais que vivenciam a experiência da infertilidade tendem a passar por profundas modificações. De acordo com Ulrich, Gagel, Hemmerling, Pastor e Kentenich (2004), com a chegada do filho, esses casais vivenciam uma dupla tarefa desenvolvimental, pois, ao mesmo tempo em que precisam realizar os ajustes necessários para receber o bebê, também experienciam mudanças em sua própria identidade decorrentes da superação da infertilidade.

Considerando que tanto a experiência da infertilidade e seu tratamento como a chegada de um bebê constituem eventos que tendem a trazer repercussões marcantes para os cônjuges, este estudo tem como objetivo apresentar uma revisão de pesquisas que investigaram o relacionamento conjugal durante a gravidez e os dois primeiros anos de vida do bebê, após concepção com o auxílio das TRA. Com isso, pretende-se fornecer subsídios à reflexão sobre um tema atual e contribuir para a realização de novos estudos.

Foram realizadas buscas nas bases de dados PsycInfo e Ovid-Medline. Descritores como assisted reproduction, reproductive technologies, in vitro fertilization e assisted reproduction foram cruzados com os termos marital, couple, family, parent e pregnancy. Na Ovid-Medline, restringiram-se as buscas por trabalhos que abordassem questões psicológicas ou psiquiátricas, uma vez que essa base compreende muitos periódicos biomédicos. Não se estabeleceram limitações quanto ao período de publicação dos estudos.

Essa busca inicial resultou na indicação de 768 artigos, os quais foram triados a partir de seus títulos e resumos para selecionarem-se apenas pesquisas empíricas que abordaram a relação de casais que obtiveram sucesso em seus tratamentos e que vivenciavam o período da gravidez ou os dois primeiros anos de vida do bebê. Estudos redigidos em idiomas que não o inglês, o espanhol ou o português não foram incluídos. De forma semelhante a Hammarberg, Fisher e Winter (2008), excluíramse pesquisas nas quais participaram especificamente casais que recorreram à doação de gametas. $\mathrm{O}$ mesmo procedimento foi adotado em relação a pesquisas exclusivas sobre gêmeos. Esses trabalhos envolvem particularidades que merecem ser discutidas em outro momento.

Realizaram-se, ainda, buscas por artigos nacionais no Index-Psi, na SciELO e no PePSIC, utilizando-se os termos "tecnologias reprodutivas" e "reprodução assistida". Excluindose artigos que tratavam exclusivamente de questões biomédicas, obtiveram-se 25 estudos nacionais. No entanto, os mesmos não se enquadraram nos critérios de inclusão deste trabalho.

Ao final desse processo, foram lidos, na íntegra, 20 artigos que abordavam o tema deste estudo. Três artigos citados nas listas de referências do material acessado foram incluídos nessa amostra.

A seguir, esses estudos serão apresentados de acordo com o período considerado. Apenas resultados e conclusões referentes à relação conjugal nesse contexto serão abordados. Após essa exposição, os estudos serão discutidos quanto a seus aspectos teóricos e metodológicos.

\section{A relação conjugal durante a gestação}

Alguns estudos investigaram o impacto do sucesso do tratamento, comparando casais que conseguiriam alcançar a gravidez com aqueles em que isso não ocorreu. Verhaak et al. (2001) realizaram um estudo longitudinal em que 207 mulheres submetidas ao seu primeiro ciclo de FIV ou ICSI foram avaliadas antes do tratamento e três semanas após o teste de gravidez, em relação a seus níveis de estresse e sua satisfação conjugal. Esta foi acessada a partir de uma escala que abordou a satisfação com a relação em geral e com a sexualidade. Observou-se que, independente de o tratamento ter obtido sucesso, houve uma queda na satisfação com a sexualidade. Os autores ressaltaram que, no período investigado, o sucesso do tratamento ainda é incerto. A partir dessa consideração, é possível pensar que, nesse momento, os casais estariam mais preocupados em confirmar a viabilidade de sua gravidez do que com sua relação.

Holter, Anderheim, Bergh e Möller (2006) realizaram um estudo semelhante, do qual participaram 117 casais, os quais foram avaliados antes, durante e após o tratamento, no que se refere ao seu bem-estar emocional, ao desejo por um filho, aos efeitos psicológicos da infertilidade e à relação conjugal. Quanto a este aspecto, os participantes responderam a duas perguntas referentes ao impacto da infertilidade sobre a relação, no que tange ao surgimento de problemas no casal e à comunicação. Essas perguntas eram respondidas através de uma escala likert de cinco pontos. De acordo com os autores, não se constataram diferenças entre os casais que engravidaram e aqueles em que isso não ocorreu. Segundo os resultados, percebeu-se o interesse, por parte dos cônjuges, em manterem a proximidade e o apoio mútuo, tanto durante o tratamento como após o mesmo. No entanto, os autores destacaram que os participantes do estudo estavam realizando sua primeira tentativa e levantaram a 
possibilidade de que o resultado poderia ser diferente para casais que passaram por repetidas frustrações.

Comparações entre casais que obtiveram sucesso no tratamento e aqueles em que isso não ocorreu também são apresentadas no estudo de Slade, Emery e Lieberman (1997). Uma amostra de 144 casais sem filhos submetidos à FIV foi avaliada no início do tratamento, duas vezes durante cada ciclo e seis meses após o término do mesmo. Os participantes responderam a escalas que avaliaram ansiedade, depressão, humor, ajustamento conjugal e satisfação sexual. De forma diversa aos estudos anteriores, os autores identificaram diferenças entre os dois grupos. Aqueles que conseguiram engravidar apresentaram uma melhora no ajustamento conjugal e na satisfação sexual, com escores mais positivos em relação a essas dimensões do que os que não obtiveram sucesso. Deve-se considerar que, neste estudo, os casais foram acessados em um intervalo maior de tempo após o término do tratamento, quando, provavelmente, a segurança em relação à gravidez já era maior.

Outros estudos compararam casais que engravidaram com o auxílio das TRA com casais que conceberam naturalmente no que se refere à conjugalidade durante a gravidez. Klock e Greenfeld (2000), por exemplo, acompanharam 74 gestantes que engravidaram a partir de procedimentos de FIV e 44 que conceberam naturalmente, com o objetivo de investigar as mudanças que essas mulheres vivenciaram durante a gravidez. A relação conjugal foi avaliada através de quatro questões que solicitaram que as participantes indicassem o quanto percebiam seu relacionamento conjugal como parceria, amizade e romance, assim como que relatassem seu grau de satisfação geral. $\mathrm{Na}$ $12^{\underline{a}}$ semana de gestação, as mães que engravidaram através da FIV relataram perceber menos seu relacionamento como uma amizade do que as mães do grupo controle. Embora sua satisfação com a relação também tenha se mostrado inferior, a média foi considerada elevada. $\mathrm{Na} 28^{\mathrm{a}}$ semana, essas diferenças não foram observadas, mas as mães que utilizaram a FIV relataram menos preocupação com o relacionamento conjugal, com a própria aparência, com o impacto econômico da gravidez e com a sua independência. As autoras atribuíram as diferenças encontradas na $12^{\mathrm{a}}$ semana a possíveis questões remanescentes da infertilidade e de seu tratamento. No entanto, destacaram que, por se tratarem de medidas de auto-relato, possíveis sentimentos negativos podem ter sido omitidos.

Harf-Kashdaei e Kaitz (2007) investigaram níveis de ansiedade e depressão, assim como os sentimentos de 30 mulheres que conceberam com o auxílio da FIV e 30 mulheres que engravidaram naturalmente, em relação a si mesmas, ao bebê e ao marido. As participantes responderam a escalas e entrevistas, que foram submetidas a análises quantitativas. As diferenças entre os grupos, no que se refere aos sentimentos pelo esposo, mostraram-se apenas marginalmente significativas, com uma tendência das mulheres do grupo FIV a demonstrarem com maior freqüência sentimentos considerados positivos. No entanto, identificou-se que os sentimentos manifestados em relação aos esposos seriam influenciados pelo fato de a causa da infertilidade ser masculina, o que, segundo os autores, demonstraria que a experiência da infertilidade apresenta repercussões mesmo após o sucesso do tratamento.
Fisher, Hammarberg e Baker (2008) investigaram diferentes aspectos do funcionamento psicossocial de 186 mulheres primíparas e não primíparas que engravidaram com o auxílio das TRA, tais como transtornos de humor, traços de personalidade, apego materno-fetal e relação conjugal. Os resultados obtidos foram comparados a dados normativos de gestantes obtidos em outros estudos. A relação conjugal foi investigada, no terceiro trimestre de gestação, a partir de uma escala que avalia duas dimensões do vínculo conjugal: cuidado e controle. Essas mulheres avaliaram seu relacionamento de forma muito positiva, descrevendo-o como mais afetuoso, sensível e companheiro, assim como menos coercitivo, crítico e dominador do que as normas populacionais disponíveis. Embora a relação conjugal tenha sido avaliada neste estudo, a mesma não consistia no foco principal do estudo, de forma que esses resultados não foram discutidos em profundidade pelos autores.

Resultados diversos foram encontrados por Stanton e Golombok (1993), que realizaram um estudo do qual participaram 15 mulheres primíparas e não primíparas que engravidaram com o auxílio da FIV e 20 que conceberam naturalmente. Em média, essas mulheres encontravam-se por volta da $30^{\underline{a}}$ semana de gestação. As participantes responderam, entre outros instrumentos, a uma escala que incluía questões sobre sua orientação social. De acordo com os autores, as mulheres que conceberam com o auxílio da FIV mostraram-se mais negativas em relação a essa dimensão de suas vidas, o que indicaria maiores dificuldades na relação conjugal e em outros relacionamentos sociais. Deve-se destacar, no entanto, que não se trata de uma escala específica para a avaliação da relação conjugal e que não se realizaram análises para identificar possíveis diferenças na avaliação da relação conjugal e dos demais relacionamentos sociais. Dessa forma, deve-se ter certa cautela ao considerar esse resultado.

Sydsjö, Wadsby, Kjellberg e Sydsjö (2002) contaram também com a participação dos maridos em seu estudo, ao investigarem a parentalidade e a relação conjugal de 110 casais que recorreram à utilização das TRAe 108 que conceberam sem o uso das mesmas. Os participantes foram acompanhados na gestação e no $1^{\circ}$ ano de vida do bebê. A relação conjugal foi avaliada com uma escala que acessou a satisfação em relação a diferentes aspectos da relação: os comportamentos do parceiro, a comunicação, a resolução de conflitos, a administração financeira, o relacionamento sexual, a criação dos filhos, as atividades de lazer, a relação com a família e os amigos, a distribuição de papéis e os valores e crenças dos parceiros. Na gravidez, ambos os grupos mostraram-se, de forma geral, satisfeitos com sua relação conjugal, havendo, no entanto, uma avaliação mais positiva por parte daqueles que recorreram às TRA em relação às seis primeiras das dimensões avaliadas. De acordo com os autores, a relação desses casais parece ter sido fortalecida em função das dificuldades vivenciadas para alcançar a gravidez. Além disso, sugeriram que, possivelmente, esses casais tenham necessitado discutir questões relativas à parentalidade e a seu relacionamento, de forma que casais que não chegaram a um acordo talvez tenham se separado antes de alcançarem a gravidez.

Hjelmstedt, Widström, Wramsby, Matthiesen e Collins (2003) compararam as respostas emocionais à gravidez de 
casais que conceberam através do uso da FIV e de casais que engravidaram naturalmente, avaliando traços de personalidade, ansiedade, atitudes quanto à gravidez, reações à infertilidade e a relação conjugal. Compuseram o primeiro grupo 55 homens e 57 mulheres que se encontravam entre $11^{\underline{a}}$ e a $17^{a}$ semanas de gestação, enquanto o segundo foi formado por 39 homens e 43 mulheres com o mesmo tempo de gravidez. A relação conjugal foi avaliada a partir de uma escala que investiga diferentes dimensões da relação, fornecendo um índice global de satisfação. Neste estudo, apenas este índice foi considerado. Não se avaliaram possíveis diferenças quanto às diversas dimensões. Não foram encontradas diferenças entre os dois grupos, sendo que as médias obtidas em ambos denotam índices elevados de satisfação conjugal. Observou-se ainda que mulheres que viviam há mais tempo com seu parceiro mostraram-se menos ansiosas em relação à saúde de seus bebês, o que, segundo os autores, poderia ser atribuído à segurança e à confiança que essas mulheres sentem em relação a seus parceiros. Dados publicados em outro artigo (Hjelmstedt, Widström, \& Collins, 2006) evidenciaram, ainda, relações entre satisfação conjugal e o vínculo estabelecido entre a mãe e seu bebê durante a $26^{\mathrm{a}}$ semana de gestação. Segundo os autores, mulheres que podem contar com um forte apoio social teriam maior possibilidade de investir emocionalmente em seus bebês. Além disso, a satisfação com a relação conjugal seria um indicador da boa capacidade dessas mulheres em estabelecer relações interpessoais, o que influenciaria a relação com o bebê.

Os achados de Ulrich et al. (2004), por sua vez, apóiam tanto a existência de semelhanças entre os dois grupos de casais como de certas dificuldades nos casais que recorrem às TRA. Os autores acompanharam 47 casais que engravidaram a partir de técnicas de FIV e 45 que conceberam naturalmente, durante o $3^{\mathrm{o}}$ trimestre de gestação e no $3^{\mathrm{o}}$ e $12^{\circ}$ meses de vida do bebê. Nos três momentos, realizou-se uma entrevista semi-estruturada com ambos os cônjuges, que foi posteriormente codificada, com o intuito de observar a satisfação conjugal, a visão do parceiro, a sexualidade, os padrões de apego do casal, a rede de apoio e a abertura na comunicação. Também foram aplicadas escalas que avaliaram a visão de si mesmo e do parceiro, a existência de queixas físicas e psicológicas, as expectativas e as percepções quanto às atividades parentais e o impacto da infertilidade. Não foram encontradas diferenças quanto à satisfação com o relacionamento, sendo que mais de $60 \%$ dos participantes de ambos os grupos declararam estar muito satisfeitos. No que se refere à sexualidade, os casais que recorreram à FIV mostraramse mais insatisfeitos sexualmente durante a gravidez do que o grupo controle, sendo que alguns desses casais chegaram a interromper sua atividade sexual por medo de machucar o bebê. As esposas que utilizaram esses procedimentos também apresentaram um padrão de apego do tipo evitativo com maior freqüência, maiores expectativas quanto ao envolvimento do marido na tarefa de acalmar o bebê e um maior índice de disfunções sexuais, que foram por elas relacionadas à decisão de ter um filho e às tentativas subseqüentes.

Resultados semelhantes em relação à sexualidade foram obtidos por Papaligoura, Panopoulou-Maratou, Solman, Arvaniti e Sarafidou (2004). Esses autores realizaram um estudo do qual participaram 30 casais que conceberam com o auxílio da ICSI, 20 casais que recorreram à FIV e 26 casais que conceberam naturalmente. Quando a criança estava com um ano, aplicou-se uma escala para avaliar o desenvolvimento do bebê e as mães responderam a entrevistas que avaliaram sua preocupação com eles. As mães que engravidaram a partir das duas TRA relataram que não tiveram relações sexuais durante a gravidez em função do medo de que algo pudesse acontecer ao bebê.

Em artigo em que foi abordado especificamente o impacto da experiência de FIV sobre os pais, Cohen, McMahon, Tennant, Saunders e Leslie (2000) acompanharam 62 homens que haviam vivenciado essa experiência e 56 homens cujas esposas conceberam naturalmente, em dois momentos: na $30^{\mathrm{a}}$ semana de gestação e quatro meses após o nascimento do bebê. Avaliaram-se os níveis de ansiedade e depressão, o apego ao feto e a relação conjugal. Esta foi acessada com o uso da Escala de Ajustamento Diádico de Spanier, que investiga quatro dimensões desse conceito - coesão, consenso, expressão de afeto e satisfação geral -, e uma escala sobre o vínculo conjugal que avalia a percepção do cuidado e do controle no relacionamento. Neste estudo, utilizou-se apenas um escore geral de ajustamento, não se diferenciando as diferentes dimensões da relação. Os autores investigaram, ainda, a influência da idade, do número de tratamentos realizados e da causa da infertilidade - masculina, feminina, mista ou desconhecida - sobre os escores obtidos nesses instrumentos. Constatou-se que, durante a gravidez, os pais que vivenciaram a experiência de FIV avaliaram seus relacionamentos de forma mais negativa, tanto no que se refere ao ajustamento conjugal como no que diz respeito à dimensão de cuidado. Segundo os autores, a chegada iminente do bebê pode exercer, sobre esses pais, um impacto que se reflete em seu relacionamento conjugal e em outros fatores, tais como sua autoestima. Não foram encontradas diferenças quanto às três variáveis intervenientes investigadas.

\section{A relação conjugal durante os dois primeiros anos de vida do bebê}

Dando continuidade ao estudo citado anteriormente (Klock \& Greenfeld, 2000), Greenfeld e Klock (2001) acompanharam as participantes dois e nove meses após o nascimento do bebê. Não foram encontradas diferenças entre os dois grupos na avaliação do relacionamento conjugal. No entanto, coerentemente com o que foi observado durante a gestação, as mulheres do grupo controle se mostraram mais preocupadas com mudanças em sua atratividade e com o efeito da parentalidade sobre sua independência.

Em estudo realizado aos quatro meses de vida do bebê, McMahon, Ungerer, Tennant e Saunders (1997) investigaram o humor, a autoestima, a adaptação à maternidade, o relacionamento mãe/filho e o ajustamento conjugal de 65 mães que engravidaram através das TRA e de 62 que conceberam naturalmente. Utilizou-se a Escala de Ajustamento Diádico de Spanier. Também foram aplicadas escalas para a avaliação da autoestima. Não se encontraram diferenças quanto à percepção do ajustamento conjugal. No entanto, as mães que recorreram às TRA apresentaram uma menor autoestima quanto à sua feminilidade e sexualidade. Os autores relacionaram este achado 
ao impacto da infertilidade, que não teria sido resolvido mesmo após o nascimento do bebê.

No artigo já mencionado, Cohen et al. (2000) relataram os dados obtidos com os maridos dessas mulheres, não constatando diferenças quanto à avaliação da relação conjugal entre o grupo FIV e o grupo controle aos quatro meses de vida do bebê. No entanto, para os pais de ambos os grupos, houve um declínio na dimensão de cuidado, o que demonstraria a existência de uma menor intimidade entre os cônjuges nesse período, corroborando, segundo os autores, os achados gerais sobre o impacto da chegada de um filho sobre o relacionamento conjugal.

Ampliando os estudos anteriores (Cohen et al., 2000; McMahon et al., 1997), Gibson, Ungerer, Tennant e Saunders (2000) acompanharam os mesmos participantes quando o bebê estava com um ano de idade. Foram utilizadas escalas que avaliaram o ajustamento conjugal, assim como a percepção de intimidade, apoio e envolvimento do parceiro. Não foram encontradas diferenças entre os dois grupos de esposas quanto ao ajustamento conjugal e à autoestima, mas os maridos que recorreram às TRA perceberam de forma mais negativa a intimidade, o apoio e o envolvimento oferecidos a eles por suas esposas, assim como apresentaram menor autoestima quando comparados ao grupo controle, de forma semelhante ao que foi observado durante a gestação. Possíveis explicações dos autores para essa constatação foram a presença de uma avaliação negativa por parte desses homens, que também apresentaram menor autoestima, ou o elevado envolvimento da esposa com a criança, que pode passar ao marido uma sensação de distanciamento. Este resultado pode ser relacionado ao constatado por Greenfeld e Klock (2001) quanto a uma menor preocupação, por parte das mulheres que engravidaram com o auxílio das TRA, em relação a outros aspectos de sua vida. Talvez essa falta de preocupação reflita, de certa forma, uma falta de atenção em relação a essas outras dimensões.

Resultados semelhantes foram encontrados por Hjelmstedt, Widström, Wramsby e Collins (2004) quanto às mudanças vivenciadas no relacionamento conjugal após o nascimento do bebê. Os autores compararam, em seu estudo, 53 homens e 55 mulheres que utilizaram as TRA e 36 homens e 40 mulheres que não passaram por essa experiência. Os participantes foram acompanhados no início da gravidez e quando o bebê estava com dois e seis meses. Utilizou-se uma escala que aborda diferentes aspectos da relação - comunicação, divisão de tarefas, atração, amizade, amor e sexualidade - para a avaliação da satisfação conjugal, obtendo-se um escore global. Os participantes que recorreram às TRA também responderam a entrevistas que investigaram a percepção da parentalidade e a intenção de recorrer novamente às TRA para alcançar uma nova gravidez. Escalas para a avaliação do estresse parental foram respondidas em ambos os grupos. Os dados foram submetidos a análises quantitativas e qualitativas. Os resultados obtidos indicaram uma queda na satisfação conjugal entre os participantes de ambos os grupos. A análise qualitativa, além de ter revelado uma grande variedade nas experiências vivenciadas, também demonstrou as dificuldades em superar a questão da infertilidade. Análises adicionais não encontraram, no entanto, relações entre a percepção da infertilidade e a satisfação conjugal. Por outro lado, observou-se que casais que se mostraram mais insatisfeitos quanto à sua relação também apresentaram maiores níveis de estresse parental. Segundo esses autores, passar pela experiência da reprodução assistida não tornaria os casais imunes aos conflitos que costumam marcar a chegada de um filho.

Por outro lado, o estudo realizado por Sydsjö et al. (2002) constatou que os casais que recorreram às TRA avaliaram todas as dimensões de seu relacionamento de forma mais positiva do que o grupo controle, exceto no que se refere à resolução de conflito. Esses casais mostraram-se, ainda, mais estáveis em suas avaliações no decorrer do primeiro ano de vida do bebê, embora tenham percebido uma mudança negativa na resolução de conflitos e positiva na divisão de tarefas. De forma geral, portanto, esses casais demonstraram satisfação em ambos os momentos analisados. Os casais do grupo controle, por outro lado, relataram uma maior queda em seus níveis de satisfação, em especial no que tange à comunicação e ao relacionamento sexual. Esses autores defendem a idéia de uma espécie de seleção dos casais no decorrer do processo de reprodução assistida. Assim, devido às demandas com que se deparam nessa trajetória, permaneceriam em tratamento apenas os casais que conseguissem lidar bem com essas questões. É importante salientar que, ao contrário do estudo do Hjelmstedt et al. (2004), em que se adotou uma medida de satisfação global, Sydsjö et al. (2002) empregaram instrumentos que avaliam separadamente a satisfação quanto a diversas dimensões da relação. Além disso, os casais foram avaliados em um período mais tardio do que no estudo anterior.

Repokari et al. (2007) acompanharam 367 casais que recorreram às TRA, comparando-os a grupo controle composto por 379 casais que conceberam naturalmente. Utilizaram-se escalas que avaliaram os níveis de depressão, a ocorrência de eventos estressantes e o histórico da infertilidade e do tratamento, além das quatro dimensões do ajustamento diádico. $\mathrm{O}$ ajustamento conjugal foi acessado somente no $2^{\mathrm{o}}$ e $12^{\mathrm{o}}$ mês de vida do bebê. Os dados obtidos pelos autores indicam um efeito neutro ou levemente positivo das TRA na avaliação da relação conjugal, levando-se em consideração as diferentes dimensões avaliadas. Os autores sugeriram, portanto, que a vivência conjunta da experiência da infertilidade pode aumentar a coesão do casal e fortalecer o relacionamento. Observaram, por outro lado, que a ocorrência de eventos estressantes esteve relacionada a uma avaliação mais negativa do consenso $\mathrm{e}$ da satisfação conjugal de mulheres que recorreram às TRA. Também encontraram que questões como o número de abortos vivenciados, no caso das mulheres, e a duração do tratamento, no caso dos homens, estiveram relacionadas a uma avaliação mais negativa de certos aspectos da relação conjugal, como satisfação e coesão. O número de tratamentos realizados mostrou-se, no entanto, um indicador de maiores índices de coesão e consenso conjugal entre as mulheres. Constatou-se, ainda, que os efeitos da infertilidade e do tratamento sobre a relação conjugal foram observados apenas durante os dois primeiros meses do bebê, o que, segundo eles, sugere que os novos desafios da parentalidade tendem a amenizar essas questões. Outras conclusões destacadas pelos autores foram o despertar de diferentes questões para homens e mulheres, a partir da experiência da infertilidade, 
e a união provocada pelo compartilhamento de experiências estressantes.

Também no que se refere às modificações percebidas no relacionamento conjugal após o nascimento do bebê, Ulrich et al. (2004) não observaram diferenças entre os dois grupos quanto aos padrões de mudança na satisfação conjugal. Uma menor porcentagem de participantes se declarou satisfeita no 3으 mês do que na gestação e no $1^{\mathrm{o}}$ ano. No entanto, mesmo no terceiro mês, mais de $60 \%$ dos participantes se declararam satisfeitos. Após o nascimento do bebê, não houve diferenças quanto à satisfação sexual entre as mulheres dos dois grupos, ao contrário do que se observou na gestação. No entanto, os homens que passaram pela FIV se mostraram mais insatisfeitos sexualmente três meses após o nascimento do bebê.

Olshansky e Sereika (2005) traçaram relações entre as modificações na satisfação conjugal e alterações nos índices de depressão, antes e após o nascimento do bebê. Participaram do estudo 25 mulheres que responderam entrevistas e escalas, que forneciam escores globais referentes às variáveis investigadas. Constatou-se uma correlação entre quedas na satisfação conjugal e aumentos nos índices de depressão. Dessa forma, os autores sugeriram que, em função da importância da relação conjugal, é possível que dificuldades na mesma sejam preditoras de depressão pós-parto.

\section{Considerações teóricas e metodológicas}

Apesar das divergências entre os estudos, é possível observar algumas tendências quanto à relação conjugal nesse contexto. A existência de diversas semelhanças entre esses casais e aqueles que engravidaram naturalmente (Hjelmstedt et al., 2004; Ulrich et al., 2004), de diferenças entre homens e mulheres quanto ao impacto provocado pela infertilidade (McMahon et al., 1997; Repokari et al., 2007) e possíveis influências sobre a sexualidade e a intimidade do casal (Cohen et al., 2000; Papaligoura et al., 2004; Ulrich et al., 2004) são questões que merecem ser destacadas. Também foi sugerido um maior envolvimento com o filho (Gibson et al., 2000) e uma menor preocupação com outras questões (Greenfeld \& Kloch, 2001; Kloch \& Greenfeld, 2000) por parte das mães que recorreram às TRA, o que poderia levar os maridos a se sentirem mais distanciados de suas esposas (Gibson et al., 2000). Tais constatações reforçam a importância de observar diferentes aspectos da relação desses casais para a compreensão de sua experiência durante a gravidez e após o nascimento do bebê.

Além disso, a revisão desses estudos também evidenciou a importância de investigar a relação conjugal nesse contexto, uma vez que a mesma mostrou-se relacionada ao apego maternofetal (Hjelmstedt et al., 2006), a índices de depressão pós-parto (Olshansky \& Sereika, 2005) e aos níveis de estresse parental (Hjelmstedt et al., 2004). A necessidade de incluírem-se os maridos nas pesquisas realizadas sobre esse tema também foi evidenciada pela sugestão de possíveis dificuldades vivenciadas por eles nesse contexto (Cohen et al., 2000; Gibson et al., 2000). Conforme demonstram Seger-Jacobs (2006) e Weiss (2006), apesar de conseguirem adotar um maior distanciamento em relação à questão da infertilidade, os homens podem se sentir marginalizados em relação ao processo e tendem a apresentar uma maior dificuldade para expressar seus sentimentos, por crerem ter a obrigação de manterem-se estáveis para poderem apoiar a esposa.

Quanto ao método empregado pelos estudos revisados, percebe-se o uso predominante de escalas e de análises quantitativas para a avaliação de variáveis específicas, como a satisfação e o ajustamento conjugal. Tal constatação corrobora o que foi pontuado por Mosmann, Wagner e Féres-Carneiro (2006), que demonstram que a maior parte dos estudos voltados à conjugalidade trabalha com os conceitos de satisfação, ajustamento e qualidade conjugal. Em uma revisão sobre o uso do conceito de satisfação conjugal, Weiss (2005) sugere algumas questões que devem ser consideradas. De acordo com esse autor, ao falarem em satisfação conjugal, os pesquisadores privilegiam o resultado em detrimento do processo, ou seja, daquilo que levou os casais a avaliarem seu relacionamento dessa forma. Portanto, visto que o relacionamento é influenciado por uma série de fatores que não agem de forma linear, seria mais interessante descrever como o relacionamento se modifica com o passar do tempo do que utilizar medidas estáticas.

Ao analisarem-se as divergências entre os resultados obtidos por esses estudos, devem-se ressaltar algumas considerações metodológicas. O primeiro fator a ser observado refere-se aos diferentes momentos em que os dados foram coletados. No estudo desenvolvido por Hjelmsted et al. (2004), por exemplo, a coleta foi realizada em momentos relativamente próximos, de forma que se poderia argumentar que os casais participantes estariam passando por um período marcado pela maior necessidade de ajustes do que aqueles que participaram do estudo de Sydsjö et al. (2002), que, após um ano, poderiam estar com suas relações mais estabilizadas.

$\mathrm{O}$ uso de diferentes instrumentos também sugere que aspectos diversos da relação conjugal estejam sendo avaliados. $\mathrm{O}$ uso de escalas que investigaram diferentes dimensões da relação, assim como a realização de entrevistas, permitiram que se avaliassem diferentes impactos da parentalidade e das TRA sobre o relacionamento conjugal. Deve-se considerar que cada um desses instrumentos fornece informações próprias, sendo que seu uso tende a gerar resultados diversos daqueles obtidos por escalas que avaliam outras dimensões da relação ou a satisfação global (Hahn \& DiPietro, 2001).

O controle de possíveis variáveis intervenientes é outro fator a ser destacado. No estudo de Kloch e Greenfeld (2000), por exemplo, embora as gestantes que recorreram à FIV fossem mais velhas e estivessem com seus companheiros há mais tempo, não houve controle quanto à idade e a duração do relacionamento, questões que podem afetar a avaliação da relação conjugal. Em alguns estudos (Fisher et al., 2008; Stanton \& Golombok, 1993), não houve controle se os casais eram primíparos, enquanto, em outros, não foram realizadas análises diferenciadas para gravidezes gemelares (Fisher et al., 2008; Greenfeld \& Klock, 2001; Klock \& Greenfeld, 2000; Ulrich et al., 2004) ou para o uso de TRA que envolviam doação de gametas (Fisher et al., 2008), o que também poderia gerar diferenças.

Percebe-se uma tendência desses estudos a privilegiarem uma comparação de tendências centrais entre grupos de casais. As poucas exceções que investigaram como outros 
aspectos poderiam influenciar as mudanças percebidas na relação conjugal ativeram-se a questões pontuais, como idade, duração do casamento, renda familiar, depressão, causa da infertilidade, transcurso do tratamento e ocorrência de eventos estressantes (Cohen et al., 2000; Harf-Kashdaei \& Kaitz, 2007; McMahon et al., 1997). Percebe-se, dessa forma, a ausência de investigações quanto aos fatores que podem favorecer ou dificultar a vivência da conjugalidade nesse período, em detrimento da simples comparação entre casais. A investigação dessas questões permitiria uma maior aproximação em relação à complexidade desse fenômeno (Belsky \& Rovine, 1990), e à própria multidimensionalidade da relação conjugal (Mosmann et al., 2006).

Dessa forma, percebe-se a falta de uma compreensão desenvolvimental a respeito do fenômeno, que considere a história de vida desses casais e de suas famílias de origem. Mesmo quando a experiência da infertilidade é investigada, não há elementos que permitam conhecer com maior profundidade aspectos referentes à relação antes da ocorrência desse evento. Dessa forma, a infertilidade tende a ser vista como um evento isolado na história daquele casal, não se considerando a influência que outras experiências vivenciadas durante o desenvolvimento da relação e dos próprios indivíduos possam exercer. Talvez uma maior compreensão a respeito desses aspectos ajudasse a explicar algumas das divergências encontradas nas pesquisas da área.

A realização de estudos que considerem os aspectos sugeridos por essas pesquisas, mas privilegiem a descrição de como os casais vivenciam esse momento em oposição à mensuração de mudanças em algumas variáveis específicas poderia contribuir para a compreensão desse contexto, ultrapassando idéias preconcebidas que ainda possam existir. Acredita-se que tanto a realização de estudos de caso, por sua abordagem detalhada e contextualizada (Robson, 1993), como a adoção de metodologias qualitativas teriam muito a colaborar para esse propósito.

\section{Referências}

Belsky, J., \& Rovine, M. (1990). Patterns of marital change across the transition to parenthood: Pregnancy to three years postpartum. Journal of Marriage and the Family, 52(1), 5-19.

Borlot, A. M. M., \& Trindade, Z. A. (2004). As tecnologias de reprodução assistida e as representações sociais de filho biológico. Estudos de Psicologia, 9(1), 63-70.

Bornholdt, E. A., Wagner, A., \& Staudt, A. C. P. (2007). A vivência da gravidez do primeiro filho à luz da perspectiva paterna. Psicologia Clínica, 19(1), 75-92.

Bouchard, G., Boudreau, J., \& Hébert, R. (2006). Transition to parenthood and conjugal life: Comparisons between planned and unplanned pregnancies. Journal of Family Issues, 27(11), 1512-1531.

Bradt, J. O. (1995). Tornando-se pais: famílias com filhos pequenos. In B. Carter \& M. McGoldrick (Orgs.), As mudanças no ciclo de vida familiar: uma estrutura para a terapia familiar (pp. 206-222). Porto Alegre: Artes Médicas.

Burns, L. H. (1987). Infertility as boundary ambiguity: one theoretical perspective. Family Process, 26, 359-372.

Burns, L. H. (2007). Psychiatric aspects of infertility and infertility treatments. Psychiatric Clinics of North America, 30(4), 689-716.
Cohen, J., McMahon, C., Tennant, C., Saunders, D., \& Leslie, G. (2000) Psychosocial outcomes for fathers after IVF conception: a controlled prospective investigation from pregnancy to four months post-partum. Reproductive Technologies, 10(3), 126-130.

Delmore-Ko, P., Pancer, S. M., Hunsberger, B., \& Pratt, M. (2000). Becoming a parent: the relation between prenatal expectations and postnatal experience. Journal of Family Psychology, 14(4), 625-640.

Fisher, J. R. W., Hammarberg, K., \& Baker, G. H. W. (2008). Antenatal mood and fetal attachment after assisted conception. Fertility and Sterility, 89(5), 1103-1112.

Gibson, F. L., Ungerer, J. A., Tennant, C. C., \& Saunders, M. (2000). Parental adjustment and attitudes to parenting after in vitro fertilization. Fertility and Sterility, 73(3), 565-574.

Greenfeld, D., \& Klock, S. C. (2001). Transition to parenthood among in vitro fertilization patients at 2 and 9 months postpartum. Fertility and Sterility, 76(3), 626-627.

Hahn, C. S., \& DiPietro, J. (2001). In vitro fertilization and the family: quality of parenting, family functioning, and child psychosocial adjustment. Developmental Psychology, 37(1), 37-48.

Hammarberg, K., Fisher, J. R. W., \& Wynter, K. H. (2008). Psychological and social aspects of pregnancy and early parenting after assisted conception: a systematic review. Human Reproduction Update, 14 (5), 395-414.

Harf-Kashdaei, E., \& Kaitz, M. (2007). Antenatal moods regarding self, baby, and spouse among women who conceived by in vitro fertilization. Fertility and Sterility, 87(6), 1306-1313.

Hjelmstedt, A, Widström, A. M., \& Collins, A. (2006). Psychological correlates of prenatal attachment in women who conceived after in vitro fertilization and women who conceived naturally. Birth, 33(4), 303-310.

Hjelmstedt, A, Widström, A. M., Wramsby, H., \& Collins, A. (2004). Emotional adaptation following successful in vitro fertilization. Fertility and Sterility, 81(5), 1254-1264.

Hjelmstedt, A., Widströn, A., Wramsby, H., Matthiesen, A., \& Collins, A. (2003). Personality factors and emotional response to pregnancy among IVF couples in early pregnancy: a comparative study. Acta Obstetricia et Gynecologica Scandinavica, 82, 152-161.

Holter, H. Anderheim, L., Bergh, C., \& Möller, A. (2006). First IVF treatment short-term impact on psychological well being and the marital relationship. Human Reproduction, 21(12), 3295-3302.

Klock, S. C., \& Greenfeld, D. A. (2000). Psychological status of in vitro fertilization patients during pregnancy: a longitudinal study. Fertility and Sterility, 73(6), 1159-1164.

Lech, M. B., \& Martins, P. C. (2003). Oscilações no desejo sexual no período gestacional. Estudos de Psicologia, 20(3), 37-46.

McMahon, C. A., Gibson, F., Leslie, G., Cohen, J., \& Tennant, C. (2003). Parents of 5-year-old in vitro fertilization children: psychological adjustment, parenting stress and the influence of subsequent in vitro fertilization treatment. Journal of Family Psychology, 17(3), 361-369.

McMahon, C. A., Ungerer, J. A., Tennant, C., \& Saunders, D. (1997). Psychosocial adjustment and the quality of the mother-child relationship at four-months postpartum after conception by in vitro fertilization. Fertility and Sterility, 68(3), 492-500.

Menezes, C. C. (2001). A relação conjugal na transição para a parentalidade: Da gestação ao segundo ano de vida do bebê. Dissertação de mestrado, Universidade Federal do Rio Grande do Sul, Porto Alegre.

Mosmann, C., Wagner, A., \& Féres-Carneiro, T. (2006). Qualidade conjugal: mapeando conceitos. Paidéia, 16(35), 315-325.

Olshansky, E., \& Sereika, S. (2005). The transition from pregnancy to postpartum in previously infertile women: a focus on depression. Archives of Psychiatry Nursing, 19(6), 273-280.

Papaligoura, Z., Panopoulou-Maratou, O., Solman, M., Arvaniti, K., \& Sarafidou, J. (2004). Cognitive development of 12 month old greek infants conceived after ICSI and the effects of the method on their parents. Human 
Reproduction, 19(6), 1488-1493.

Perren, S., Wyl, A. V., Bürgin, D., Simoni, H., \& Klitzing, K. V. (2005). Intergenerational transmission of marital quality across the transition to parenthood. Family Process, 44(4), 441-459.

Piccinini, C. A., Gomes, A. G., Nardi, T. D., \& Lopes, R. S. (2008). Gestação e a constituição da maternidade. Psicologia em Estudo, 13(1), 63-72.

Pittman, F. S. (1987). Turning points: treating families in transition and crisis. New York: W. W. Norton \& Company.

Repokari, I., Punamäki, R. I., Unkila-Kallio, I., Vilska, S., Poikkeus, P. P., Sinkkonem, J., Almqvist, F., Tiitnem, A., \& Tulppala, A. (2007). Infertility treatment and marital relationships: a 1-year prospective study among successfully treated ART couples and their controls. Human Reproduction, 22(5), 1481-1491.

Robson, C. (1993). Real world research: A resource for social sciences and practioner researcher. Oxford: Blackwell.

Savitz-Smith, J. (2003). Couples undergoing infertility treatment: implications for counselors. The Family Journal, 11, 383-387.

Seger-Jacob, L. (2006). Estresse na gênese e no tratamento da infertilidade. In R M. M. Melamed \& J. Quayle (Orgs.), Psicologia em reprodução assistida: experiências brasileiras (pp. 121-153). São Paulo: Casa do Psicólogo.

Slade, P., Emery, J., \& Lieberman, B. A. (1997). A prospective, longitudinal study of emotions and relationships in in-vitro fertilization treatment. Human Reproduction, 12(1), 183-190.

Spotorno, P. M., Silva, I. M., \& Lopes, R. C. S. (2008). Expectativas e sentimentos de mulheres em situação de reprodução medicamente assistida. Aletheia, 28, 104-118.

Stanton, F., \& Golombok, S. (1993). Maternal-fetal attachment during pregnancy following in vitro fertilization. Journal of Psychosomatic Obstetrics Gynaecology, 14, 153-158.

Stern, D. N. (1997). A constelação da maternidade: o panorama da psicoterapia pais/bebê. Porto Alegre: Artes Médicas.

Sydsjö, G, Wadsby, M., Kjellberg, S., \& Sydsjo, A. (2002). Relationships and parenthood in couples after assisted reproduction and in spontaneous primiparous couples: a prospective long-term follow-up study. Human Reproduction, 17(12), 3342-3250.

Ulrich, D., Gagel, D. E., Hemmerling, A., Pastor, V. S., \& Kentenich, H. (2004). Couples becoming parents: something special after IVF? Journal of Psychosomatic Obstetrics \& Gynecology, 25, 99-113.

Verhaak, C., Smeenk, J., Eugster, A., Minnen, A., Kremer, J., \& Kraaimaat, F. (2001). Stress and marital satisfaction among women before and after their first cycle of in vitro fertilization and intracytoplasmatic sperm injection. Fertility and Sterility, 76(3), 525-531.

Weiss, R. L. (2005). A critical view of marital satisfaction. In W. M. Pinsof (Org.), Family Psychology: the art of the science (pp. 23- 41). Cary: Oxford University Press.

Weiss, T. K. (2006). O impacto da infertilidade e seu tratamento nos casais. In R. M. M. Melamed \& J. Quayle (Orgs.), Psicologia em reprodução assistida: experiências brasileiras (pp. 105-119). São Paulo: Casa do Psicólogo.

Isabela Machado da Silva, Mestre em Psicologia pela Universidade Federal do Rio Grande do Sul (UFRGS), é doutoranda em Psicologia na Universidade Federal do Rio Grande do Sul (UFRGS) e bolsista de doutorado CAPES. Endereço para correspondência: Universidade Federal do Rio Grande do Sul, Instituto de Psicologia - Núcleo de Infância e Família (NUDIF), Rua Ramiro Barcelos, 2600, sala 108 - Bairro Santa Cecília, CEP. 90035-003 Porto Alegre-RS. Telefone: (51)3308-5145. E-mail: isabela.ms@gmail.com Rita de Cássia Sobreira Lopes, doutora em Psicologia pela Universidade de Londres (UL), é professora do Programa de Pós-Graduação em Psicologia da Universidade Federal do Rio Grande do Sul. E-mail: sobreiralopes@portoweb.com.br 